International Mathematical Forum, 2, 2007, no. 35, 1755 - 1761

\title{
A Note on the Weakly Quasi-Convex Functions
}

\author{
S. Lahrech \\ Dept. of Mathematics, Faculty of Science, Mohamed first University \\ Oujda, Morocco, (GAFO Laboratory) \\ A. Jaddar \\ National School of Managment, Mohamed first University \\ Oujda, Morocco, (GAFO Laboratory)

\section{J. Hlal} \\ Dept. of Mathematics, Faculty of Science, Mohamed first University
} Oujda, Morocco, (GAFO Laboratory)

\section{A. Ouahab}

Dept. of Mathematics, Faculty of Science, Mohamed first University Oujda, Morocco, (GAFO Laboratory)

\begin{abstract}
A. Mbarki
National School of Applied Sciences, Mohamed first University Oujda, Morocco, (GAFO Laboratory)
\end{abstract}

\begin{abstract}
The main purpose of this paper is to characterize weakly quasiconvex functions via the limiting subdifferential.
\end{abstract}

Mathematics Subject Classification: 49J52, 49J50

Keywords: Mean value Theorem, Weakly quasi-convex functions, Limiting subdifferential

\section{Introduction}

The generalized convexity have found extensive applications in areas such as mathematical programming and economics. Such notion is a natural gen- 
eralization of convexity. In particular many results have been done for the weak convexity, we can cite for instance the result given in [4] by Jourani and Théra which used this notion (with modulus $\varepsilon$ ) in order to characterize the $\varepsilon$-monotonicity of the limiting Fréchet $\varepsilon$-subdifferential by adopting the following definition :

A real function $f$ defined on a normed space $(X,\|\|$.$) is said to be weakly$ convex (with modulus $\varepsilon>0$ ) or $\varepsilon$-convex if for all $x, y \in X$ and $\lambda \in[0,1]$ we have :

$$
f(\lambda x+(1-\lambda) y) \leq \lambda f(x)+(1-\lambda) f(y)+\varepsilon \lambda(1-\lambda)\|x-y\| .
$$

Our aim in this paper is to extend this definition to a big class called the weak quasi-convexity (with modulus $\varepsilon>0$ ) in order to characterize it via the limiting subdifferential.

Recall that a real function $f$ defined on a normed space $(X,\|\|$.$) is said to be$ weakly quasi-convex (with modulus $\varepsilon>0$ ) or $\varepsilon$-quasi-convex if for all $x, y \in X$ and $\lambda \in[0,1]$ we have :

$$
f(\lambda x+(1-\lambda) y) \leq \max \{f(x), f(y)\}+\varepsilon \lambda(1-\lambda)\|x-y\| .
$$

We can easily see that any weakly convex function is weakly quasi-convex.

The rest of this paper is organized as follows. After recalling some definitions and properties in section 2 we give in section 3 a necessary and sufficient conditions for a function $f$ to be weakly quasi-convex.

\section{Basic definitions and properties}

In this section we recall several definitions and results necessary for further developments.

Throughout this paper, $(X,\|\|$.$) denotes a reflexive separable Banach space,$ $X^{*}$ its topological dual, $B^{*}$ the closed unit ball in $X^{*}$.

Under the above conditions, $(X,\|\cdot\|)$ is an Asplund space. Moreover, $B^{*}$ is metrizable for the weak star topology $* \sigma\left(X^{*}, X\right)$ of $X^{*}$.

Let $f: X \longrightarrow R \cup\{+\infty\}$ be an extended-real valued function. We recall that: $\operatorname{dom} f=\{x \in X / f(x)<+\infty\}, x \stackrel{f}{\rightarrow} \bar{x}$ (respectively, $\stackrel{\omega^{*}}{\rightarrow}$ ) means that $x \rightarrow \bar{x}$ with $f(x) \rightarrow f(\bar{x})$ ( respectively, the convergence for the weak-star topology of $\left.\mathrm{X}^{*}\right)$.

Definition 2.1 Let $f: X \longrightarrow R \cup\{+\infty\}$ be an extended-real valued function and let $\varepsilon \geq 0$.

The Fréchet $\varepsilon$-subdifferential of $f$ at $x \in \operatorname{dom} f$ is defined by:

$$
\partial_{\varepsilon}^{F} f(x)=\left\{x^{*} \in X^{*} / \liminf _{u \rightarrow x} \frac{f(u)-f(x)-\left\langle x^{*}, u-x\right\rangle}{\|u-x\|} \geq-\varepsilon\right\} .
$$


The limiting Fréchet $\varepsilon$-subdifferential at $x \in \operatorname{dom} f$ is defined by:

$$
\hat{\partial}_{\varepsilon} f(x)=\limsup _{\substack{f \\ y}} \partial_{\varepsilon}^{F} f(y),
$$

where"limsup" stands the sequential Kuratowski-Painlevé upper limit with respect to the strong topology of $X$ and the weak-star topology of $X *$, i.e.:

$\lim \sup \partial_{\varepsilon}^{F} f(y)=\left\{x^{*} \in X^{*} / \exists x_{n} \stackrel{f}{\rightarrow} x, \exists x_{n}^{*} \stackrel{\omega^{*}}{\rightarrow} x^{*}\right.$ such that $\left.x_{n}^{*} \in \partial_{\varepsilon}^{F} f\left(x_{n}\right) \forall n \in N\right\}$. $y \stackrel{f}{\rightarrow} x$

When $\varepsilon=0$, then the set $(2)$ is denoted by $\hat{\partial} f(x)$.

Since $\mathrm{X}$ is an Asplund space, then we have $($ see $[3,6])$ :

$$
\hat{\partial}_{\varepsilon} f(x)=\hat{\partial} f(x)+\varepsilon B^{*} .
$$

Recall also (see [5]) that if the function $f$ is l.s.c. around $x \in \operatorname{dom} f$, then the limiting subdifferential (in the Mordukhovich sense) is given by:

$$
\partial f(x)=\limsup _{\substack{f \\ y, x, \varepsilon \backslash 0}} \partial_{\varepsilon}^{F} f(y) .
$$

Proposition 2.2 [1] Let $f: X \longrightarrow R \cup\{+\infty\}$ be an extended-real valued function and let $x \in \operatorname{dom} f$. Then we have:

$$
\partial^{F} f(x) \subset \partial^{D^{+}} f(x) \subset \partial^{C} f(x),
$$

where $\partial^{D^{+}} f(x)$ is the Dini upper subdifferential and $\partial^{C} f(x)$ is the Clarke subdifferential of $f$ at $x$.

\section{Characterization of weakly quasi-convex func- tions via the limiting subdifferential}

We give in this section a necessary and sufficient conditions for a function $f$ to be weakly quasi-convex using the limiting subdifferential.

Let us start first with the necessary condition.

Proposition 3.1 Let $f: X \longrightarrow R$ be a l.s.c real function.

If $f$ is weakly quasi-convex (with modulus $\varepsilon>0$ ), then for all $x, y$ in $X$ the following implication holds :

$$
\left[\exists x^{*} \in \partial f(x):\left\langle x^{*}, y-x\right\rangle>\varepsilon\|y-x\|\right] \Rightarrow f(z) \leq f(y)+\varepsilon \lambda(1-\lambda)\|x-y\|,
$$

for any $z=\lambda x+(1-\lambda) y$ with $\lambda \in[0,1]$. 
Proof. Let $x, y \in X$ and $x^{*} \in \partial f(x)$ such that $\left\langle x^{*}, y-x\right\rangle>\varepsilon\|y-x\|$.

Then, there exists $\eta>0$ such that $\left\langle x^{*}, y-x\right\rangle>\varepsilon\|y-x\|+\eta$. Since $x^{*} \in \partial f(x)$ then, by (4) there exists sequences $x_{n} \stackrel{f}{\rightarrow} x, x_{n}^{*} \stackrel{\omega^{*}}{\rightarrow} x^{*}, \varepsilon_{n} \searrow 0$ satisfying $x_{n}^{*} \in \partial_{\varepsilon_{n}}^{F} f\left(x_{n}\right)$ for any $n \in N$. By the continuity of $u \longmapsto\|y-u\|$ and $\langle.,$. there is $n_{0} \in N$ such that $\forall n \geq n_{0} \quad \forall \varepsilon^{\prime}$ close to $\varepsilon\left(\varepsilon^{\prime}>\varepsilon\right)$ we have:

$$
\left\langle x_{n}^{*}, y-x_{n}\right\rangle>\varepsilon^{\prime}\left\|y-x_{n}\right\|+\eta \text {. }
$$

On the other hand, $x_{n}^{*} \in \partial_{\varepsilon_{n}}^{F} f\left(x_{n}\right) \subset \hat{\partial}_{\varepsilon_{n}} f\left(x_{n}\right)$. Consequently, by virtue of (3), there exists $\tilde{x}_{n}^{*} \in \hat{\partial} f\left(x_{n}\right)$ such that $x_{n}^{*}=\tilde{x}_{n}^{*}+k_{n}^{*}$ with $\left\|k_{n}^{*}\right\|_{X^{*}} \leq \varepsilon_{n}$. From (5) we get:

$\forall n \geq n_{0} \forall \varepsilon^{\prime}$ close to $\varepsilon\left(\varepsilon^{\prime}>\varepsilon\right)\left\langle\tilde{x}_{n}^{*}, y-x_{n}\right\rangle>\varepsilon^{\prime}\left\|y-x_{n}\right\|-\left\langle k_{n}^{*}, y-x_{n}\right\rangle+\eta$.

Therefore,

$$
\forall n \geq n_{0} \forall \varepsilon^{\prime} \text { close to } \varepsilon\left(\varepsilon^{\prime}>\varepsilon\right)\left\langle\tilde{x}_{n}^{*}, y-x_{n}\right\rangle>\left(\varepsilon^{\prime}-\varepsilon_{n}\right)\left\|y-x_{n}\right\|+\eta .
$$

Let $\varepsilon^{\prime}>\varepsilon$ such that

$$
\forall n \geq n_{0}\left\langle\tilde{x}_{n}^{*}, y-x_{n}\right\rangle>\left(\varepsilon^{\prime}-\varepsilon_{n}\right)\left\|y-x_{n}\right\|+\eta .
$$

By virtue of (7) and taking into account that $\tilde{x}_{n}^{*} \in \hat{\partial} f\left(x_{n}\right)$, we deduce that there exists $\tilde{x}_{n, m}^{*} \stackrel{\omega^{*}}{\rightarrow} \tilde{x}_{n}^{*}, x_{n, m} \stackrel{f}{\rightarrow} x_{n}$ with $\tilde{x}_{n, m}^{*} \in \partial^{F} f\left(x_{n, m}\right) \subset \partial^{D^{+}} f\left(x_{n, m}\right)$ such that

$\forall n \geq n_{0} \exists m_{0} \forall m \geq m_{0}$ we have

$$
f^{D^{+}}\left(x_{n, m}, y-x_{n, m}\right) \geq\left\langle\tilde{x}_{n, m}^{*}, y-x_{n, m}\right\rangle>\left(\varepsilon^{\prime}-\varepsilon_{n}\right)\left\|y-x_{n, m}\right\|+\eta,
$$

where $f^{D^{+}}\left(x_{n, m}, y-x_{n, m}\right)=\limsup _{t \searrow 0} \frac{f\left(x_{n, m}+t\left(y-x_{n, m}\right)\right)-f\left(x_{n, m}\right)}{t}$.

Consequently, there are sequences $\left(s_{n}\right)$ and $\left(m_{r}\right)$ of positive integers satisfying the following conditions:

$$
x_{s_{n}, m_{r}} \stackrel{f}{\rightarrow} x \text { as } n, r \rightarrow+\infty ; m_{r} \rightarrow+\infty \text { as } r \rightarrow+\infty ; s_{n} \rightarrow+\infty \text { as } n \rightarrow+\infty
$$

and for every positive integers $n, r$

$f^{D^{+}}\left(x_{s_{n}, m_{r}}, y-x_{s_{n}, m_{r}}\right) \geq\left\langle\tilde{x}_{s_{n}, m_{r}}^{*}, y-x_{s_{n}, m_{r}}\right\rangle>\left(\varepsilon^{\prime}-\varepsilon_{s_{n}}\right)\left\|y-x_{s_{n}, m_{r}}\right\|+\eta$.

Consequently, there exists a sequence $\tau_{n, r} \searrow 0$ and there is an integer $n_{2}$ such that $\forall n, r \geq n_{2}$

$$
f\left(x_{s_{n}, m_{r}}+\tau_{n, r}\left(y-x_{s_{n}, m_{r}}\right)\right)>f\left(x_{s_{n}, m_{r}}\right)+\tau_{n, r}\left(1-\tau_{n, r}\right)\left(\varepsilon^{\prime}-\varepsilon_{s_{n}}\right)\left\|y-x_{s_{n}, m_{r}}\right\| .
$$


Since $\varepsilon^{\prime}>\varepsilon$, then for $n, r$ large enough we obtain

$$
f\left(x_{s_{n}, m_{r}}+\tau_{n, r}\left(y-x_{s_{n}, m_{r}}\right)\right)>f\left(x_{s_{n}, m_{r}}\right)+\tau_{n, r}\left(1-\tau_{n, r}\right) \varepsilon\left\|y-x_{s_{n}, m_{r}}\right\| .
$$

Hence, by virtue of the weak quasi-convexity of the function $f$ it follows $\max \left(f\left(x_{s_{n}, m_{r}}\right), f(y)\right)=f(y)$ for $n, r$ large enough. On the other hand, $x_{s_{n}, m_{r}} \stackrel{f}{\rightarrow}$ $x$. Therefore, $\max (f(x), f(y))=f(y)$ and the result follows.

The sufficient condition is given by the following proposition.

Proposition 3.2 Let $f: X \longrightarrow R$ be a locally Lipschitzian real-function and let $\varepsilon>0$. If for all $x, y \in X$ the following implication holds :

$$
\exists x^{*} \in \partial f(x):\left\langle x^{*}, y-x\right\rangle>\varepsilon\|y-x\| \Rightarrow f(z) \leq f(y)+\varepsilon \lambda(1-\lambda)\|x-y\|,
$$

for any $z=\lambda x+(1-\lambda) y$ with $\lambda \in[0,1]$. Then $f$ is weakly quasi-convex (with modulus $2 \varepsilon$ ).

Proof. Assume the contrary. Then there are $x, y \in X$ and $z=\lambda x+(1-\lambda) y \in$ ]$x, y[,(\lambda \in] 0,1[)$ such that

$$
f(z)>\max \{f(x), f(y)\}+2 \varepsilon \lambda(1-\lambda)\|x-y\| .
$$

Assume that $\lambda \in\left[\frac{1}{2}, 1[\right.$. Applying [Theorem 18, [4]] at points $x, z$, we deduce that there are sequences $\left.c_{n} \rightarrow c \in\right] x, z\left[\right.$ and $c_{n}^{*} \in \hat{\partial}_{\frac{1}{n}} f\left(c_{n}\right)$ satisfying

$$
\liminf _{n \rightarrow \infty}\left\langle c_{n}^{*}, z-c_{n}\right\rangle \geq \frac{f(z)-f(x)}{\|z-x\|}\|z-c\| .
$$

Since $c_{n}^{*} \in \hat{\partial}_{\frac{1}{n}} f\left(c_{n}\right)$, then there exists $\tilde{c}_{n}^{*} \in \hat{\partial} f\left(c_{n}\right)$ such that: $c_{n}^{*}=\tilde{c}_{n}^{*}+k_{n}^{*}$ with $\left\|k_{n}^{*}\right\| \leq \frac{1}{n}$. Fix an $\eta>0$ such that

$$
f(z)>\max \{f(x), f(y)\}+2 \varepsilon \lambda(1-\lambda)\|x-y\|+\eta \frac{\|z-x\|}{\|z-c\|} .
$$

Then, since $k_{n}^{*} \rightarrow 0$ for the strong topology of $X^{*}$ and using the fact that

$$
\liminf _{n \rightarrow \infty}\left\langle c_{n}^{*}, z-c_{n}\right\rangle \geq \frac{f(z)-f(x)}{\|z-x\|}\|z-c\|
$$

we deduce that there exists an integer $n_{0}$ such that for all $n \geq n_{0}$ :

$$
\left\langle\tilde{c}_{n}^{*}, z-c_{n}\right\rangle \geq \frac{f(z)-f(x)}{\|z-x\|}\left\|z-c_{n}\right\|-\eta .
$$


Let us prove that there exists $c^{*} \in \partial f(c)$ such that $\left\langle c^{*}, y-c\right\rangle>\varepsilon\|y-c\|$. It is clear that there exist $\varepsilon^{\prime}>\varepsilon$ and an integer $n_{1}$ such that for all $n \geq n_{1}$ :

$$
\frac{f(z)-f(x)}{\|z-x\|}>\frac{2 \varepsilon^{\prime} \lambda(1-\lambda)\|x-y\|}{\|z-x\|}+\frac{\eta}{\left\|z-c_{n}\right\|} .
$$

By virtue of (9), (10) and from $\|z-x\|=(1-\lambda)\|y-x\|$ with $\lambda \geq \frac{1}{2}$ we can easily see that:

$$
\left\langle\tilde{c}_{n}^{*}, z-c_{n}\right\rangle>\varepsilon^{\prime}\left\|z-c_{n}\right\|, \text { for } n \text { large enough. }
$$

On the other hand, $\tilde{c}_{n}^{*} \in \hat{\partial} f\left(c_{n}\right)$, then by (4) there are sequences $c_{n, m}^{*} \stackrel{\omega^{*}}{\rightarrow} \tilde{c}_{n}^{*}$ $(\forall n), c_{n, m} \stackrel{f}{\rightarrow} c_{n}(\forall n)$, as $m \rightarrow+\infty$ satisfying $c_{n, m}^{*} \in \partial^{F} f\left(c_{n, m}\right)$ for any integers $m$ and $n$.

By Proposition 2, $c_{n, m}^{*} \in \partial^{C} f\left(c_{n, m}\right)(\forall m, n)$. On the other hand, $f$ is locally Lipschitzian around $c$. Hence, there is $K>0$ ( $K$ the Lipschitz constant of $f$ at $c$ ), there are sequences $\left(s_{n}\right)$ and $\left(m_{r}\right)$ of positive integers and there is a positive integer $n_{2}$ such that

$$
c_{s_{n}, m_{r}} \stackrel{f}{\rightarrow} c \text { as } n, r \rightarrow+\infty, \forall n, r \geq n_{2} \quad \partial^{C} f\left(c_{s_{n}, m_{r}}\right) \subset K B^{*} .
$$

Moreover,

$$
m_{r} \rightarrow+\infty \text { as } r \rightarrow+\infty, s_{n} \rightarrow+\infty \text { as } n \rightarrow+\infty .
$$

Since for every integer $n$

$$
c_{s_{n}, m_{r}}^{*} \stackrel{\omega^{*}}{\rightarrow} \tilde{c}_{s_{n}}^{*} \text { as } r \rightarrow+\infty,
$$

then for $n$ large enough

$$
\left\|\tilde{c}_{s_{n}}^{*}\right\| \leq \liminf _{r \rightarrow \infty}\left\|c_{s_{n}, m_{r}}^{*}\right\| \leq k .
$$

Consequently, $\left(\tilde{c}_{s_{n}}^{*}\right)_{n}$ is bounded in $X^{*}$. Since $X$ is separable, then without loss of generality, we can assume that there is $c^{*} \in X^{*}$ such that $\tilde{c}_{s_{n}}^{*} \stackrel{\omega^{*}}{\rightarrow} c^{*}$. On the other hand, $K B^{*}$ is metrizable for the weak star topology $* \sigma\left(X^{*}, X\right)$. Therefore, we can assume that

$$
c_{s_{n}, m_{r}}^{*} \stackrel{\omega^{*}}{\rightarrow} c^{*} \text { as } n, r \rightarrow+\infty .
$$

By (11), it is easy to see that there is a sequence $\left(r_{n}\right)_{n}$ satisfying $r_{n} \rightarrow+\infty$ and for $n$ large enough

$$
\left\langle c_{s_{n}, m_{r_{n}}}^{*}, z-c_{s_{n}, m_{r_{n}}}\right\rangle>\varepsilon^{\prime}\left\|z-c_{s_{n}, m_{r_{n}}}\right\| .
$$

Consequently, tending $n \rightarrow \infty$ we obtain :

$$
\left\langle c^{*}, z-c\right\rangle \geq \varepsilon^{\prime}\|z-c\| .
$$


On the other hand, $(z-c)=\frac{\|z-c\|}{\|y-c\|}(y-c)$ and $\varepsilon^{\prime}>\varepsilon$. Hence, $\left\langle c^{*}, y-c\right\rangle>$ $\varepsilon\|y-c\|$ with $c^{*} \in \partial f(c)$. Therefore, using the fact that $z=\mu c+(1-\mu) y$, where $\mu=\lambda \frac{\|y-x\|}{\|y-c\|}$, it follows

$$
f(z) \leq f(y)+\varepsilon \mu(1-\mu)\|c-y\| .
$$

In this case,

$$
f(z) \leq f(y)+\varepsilon \lambda \frac{\|y-x\|}{\|y-c\|}\left(\frac{\|y-c\|-\lambda\|y-x\|}{\|y-c\|}\right)\|y-c\| .
$$

Since $\|y-c\| \leq\|y-x\|$, then

$$
f(z) \leq f(y)+\varepsilon \lambda(1-\lambda)\|y-x\| \frac{\|y-x\|}{\|y-c\|} .
$$

On the other hand, $\frac{\|y-x\|}{\|y-c\|}=\frac{\mu}{\lambda} \leq 2\left(\lambda \geq \frac{1}{2}\right)$. Consequently,

$$
f(z) \leq f(y)+2 \varepsilon \lambda(1-\lambda)\|y-x\|,
$$

which contradicts $(7)$.

In the case where $\lambda \in] 0, \frac{1}{2}[$, we use the same arguments by applying [Theorem 18, [4]] at points $z, y$. Thus, we arrive at $f(z) \leq f(x)+2 \varepsilon \lambda(1-\lambda)\|y-x\|$, which contradicts also (7). Thus, we achieve the proof.

\section{References}

[1] Aussel. D. Mean Value Theorem and Generalized Convexity in Nonsmooth Analysis. These, Université Blaise Pascal, (1994).

[2] Brezis. H. Analyse fonctionnelle, théorie et Applications. Masson. Paris. (1983).

[3] Jofré. A, Luc. D. T, Théra. M. $\varepsilon$-subdifferential and $\varepsilon$ - monotonicity, Nonlinear Analysis, Th. Meth. and Appl., 33, (1998), 71-90.

[4] Jourani. A, Théra. M. On Limiting Fréchet $\varepsilon$-Subdifferentials. Generalized convexity generalized monotonicity: recent results (Luminy 1996), Nonconvex Optim. Appl, (1997),185-198.

[5] Kruger. A. Y. Properties of Generalized Differentials, Siberian Math. J. 26, (1985), 822-832.

[6] Mordukhovich. B. S, Shao. Y. Nonsmooth sequential analysis in Asplund spaces, Trans. Amer. Math. Soc., 348, (1996), 1235-1280.

Received: November 18, 2006 